\title{
Multiturn and high precision through-shaft magnetic sensors
}

\author{
Dr. Gerald Masson, Yannick Ronnat, Thierry Dorge, Dr. Stephan Biwersi* \\ Moving Magnet Technologies SA \\ 1, Rue Christiaan Huygens, 25000 Besançon, France - www.movingmagnet.com \\ $\left(^{*}\right)$ : corresponding author - sbiwersi@movingmagnet.com
}

\begin{abstract}
In this paper, we will present new applications of MMT's through shaft $360^{\circ}$ sensor technology to higher precision or multi-turn applications.

\section{Introduction}

Magnetic sensors have several advantages which make them attractive for automotive applications: they are contactless (there is no mechanical wear-out), insensitive to dirt and are relatively simple. MMT formerly developed a $360^{\circ}$ through shaft angular position sensor technology using a probe which measures the magnetic field angle variation generated by a rotary diametrically magnetized magnet. Now, MMT is focusing on derived designs providing additional features like a multi-turn output, an improved accuracy or a very low sensitivity to external magnetic fields.

One of the targets is to meet the standard requirements for the automotive steering applications (the Electronic Stability Program legislation being one of the key drivers), and especially an accuracy of $+/-1^{\circ}$ or below. An other aim is to provide an absolute multi-turn output in a compact embodiment.

In this paper, after presenting the basics of our $360^{\circ}$ through shaft solution we will introduce a generic principle to improve the sensor linearity and provide prototype measurement results.

Then, we will present absolute multi-turn sensor concepts including again prototype design and measurement results.
\end{abstract}

\section{Field rotation magnetic position sensors}

Magnetic analog position sensors using a moving permanent magnet have witnessed a significant evolution over the last years. Indeed, new ICs (integrated circuits) [1,2,3] with ability to measure the rotation of the magnetic field have become available on the market and propose an interesting alternative to position sensors (field amplitude measurement) which were the standard until then [4].

A typical application of these new components is described in Figure 1. A diametrically magnetized disk magnet is rotating in front of an IC measuring in a "single point" two components of the magnetic field in a plane parallel to the magnet.

These two magnetic field components have a sine and cosine profile over the magnet rotation. Therefore, through the use of the Arctan function and an appropriate algorithm, it is possible to deduce the absolute position of the magnet along a complete turn. Ideally, the signal processing is directly integrated in the IC.

Such sensor designs provide a significant number of advantages as compared to previous field amplitude measurement sensors. Indeed, their structure is really simple (no ferromagnetic flux collecting part), they feature no magnetic hysteresis, are much less sensitive to temperature or geometrical tolerances, operate with higher airgap and are very accurate.

Several angular sensors using this principle are now on the market. Variants using for example a small disk magnet magnetized in its thickness are also available for short stroke linear sensing (typically < $20 \mathrm{~mm})$.

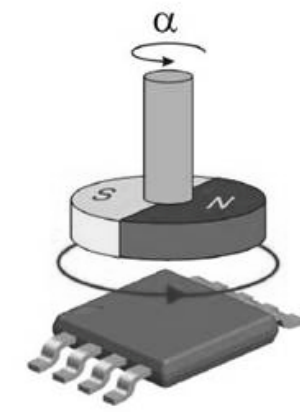

Figure 1 : end of shaft $360^{\circ}$ magnetic sensor principle

\section{Through shaft contactless $360^{\circ}$ magnetic sensor structure}

Although principles described hereabove provide attractive and efficient solutions for many applications, several challenges were still left open like the ability to provide a $360^{\circ}$ (or less) output in a through shaft mechanical configuration or a compact long stroke linear position sensor (typically $>20 \mathrm{~mm}$ ). 
MMT developed over the last years several solutions to overcome these challenges for rotary and linear sensors $[5,6]$. We will focus here on through shaft $360^{\circ}$ rotary sensors for whom MMT's solution is described in Figure 2.

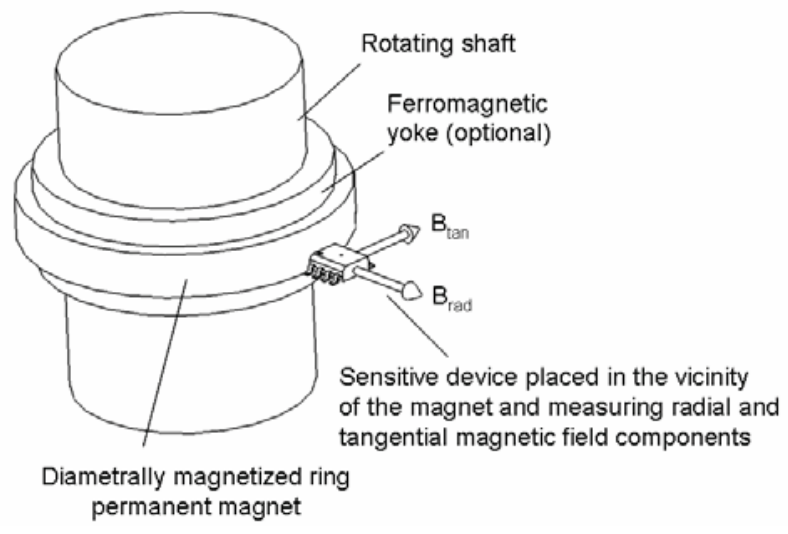

Figure 2 : through shaft $360^{\circ}$ position sensor principle

In the proposed design the IC measuring the magnetic field is installed next to a diametrically magnetized magnet ring. In this case, it is relevant that the angle of the magnetic field does not follow the rotational angle of the shaft due to the fact that although magnetic field components theoretically have a sine and cosine shape, they do not have the same amplitude. It has been shown however [7] that through the use of a compensation parameter before the computation of the field angle, this difference can be balanced and that an accurate output can be reached for various sizes and strokes of sensor. Figure 3 provides the example of a steering angle sensor prototype with its measurement curve over the $40 /+150^{\circ} \mathrm{C}$ temperature range. As one can see, the linearity is below $+/-0.5 \%$ of the full stroke.
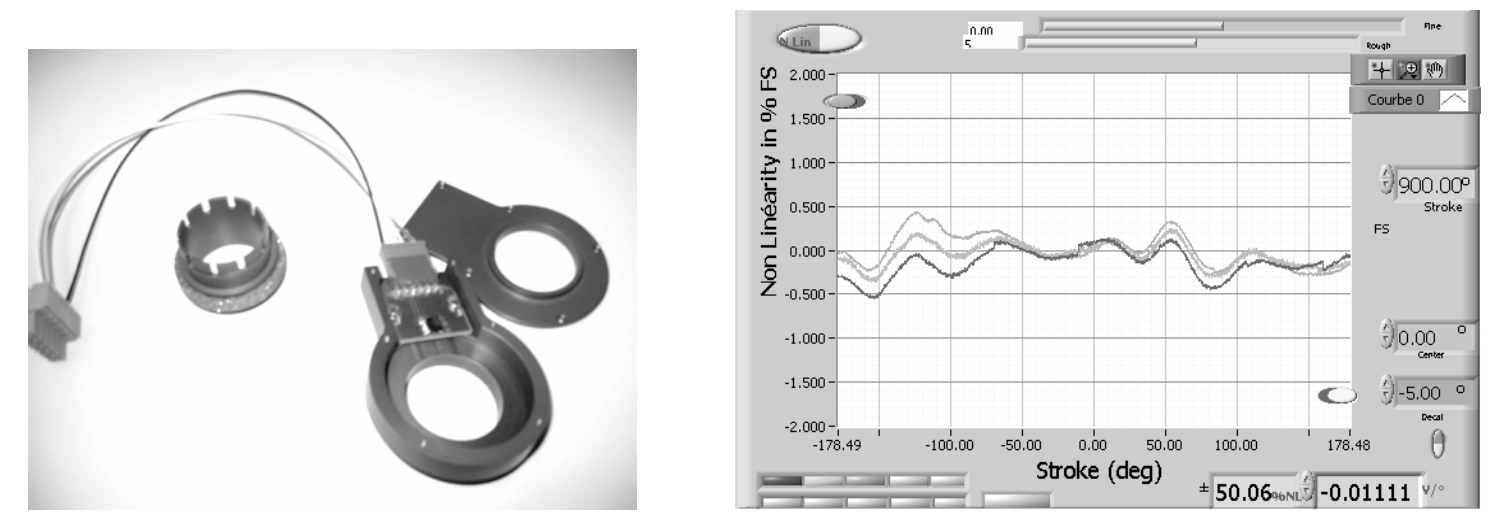

Figure 3 : steering angle sensor prototype and measurement curves

\section{Improved accuracy sensor}

As stated in the previous paragraph, MMT's expertise in the field of magnetization and programming now makes it possible to reach accuracies of $+/-0.5 \%$ of the full stroke taking into account various manufacturing parameters. However, some applications (as for example some steering angle or motor position sensors) are much more demanding and require significantly better performances.

Considering that, and in order to improve the accuracy, it is important to understand the main reasons for non linearity.

In that type of sensor, magnetization obviously plays an important role. Indeed, as a permanent magnet has a relative permeability above 1 (typically from 1.05 to 1.25) it doesn't exactly behave as air. Therefore, the field lines generated by the magnetizing coil will be slightly affected when crossing the magnet and will not follow a perfect straight line (Figure 4).

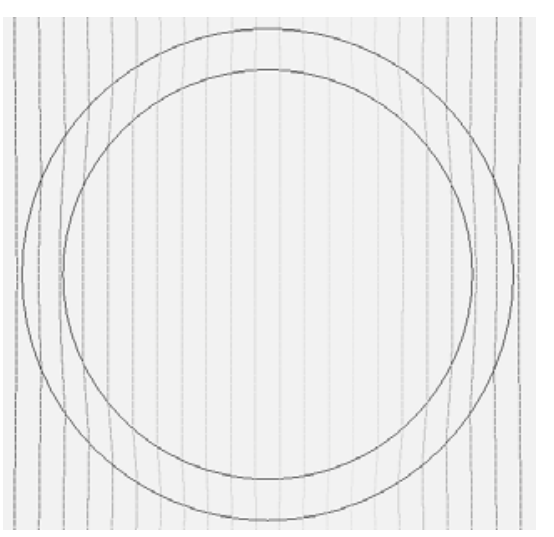

Figure $4: 2 D$ simulation of field lines distortion in a diametrically magnetized magnet 
As a consequence, the magnetization will not be perfectly diametrical, which will result in harmonics distorting the sine and cosine shape of the field components (Figure 5). Especially, one will witness a strong content of third harmonics (Figure 6). Although part of this default can be handled through careful programming, it can't be completely balanced in a single sensor and will therefore result in an accuracy error that has to be considered if one wants to reach very good accuracies. Therefore, taking this default into account is the key for a better sensor performance.

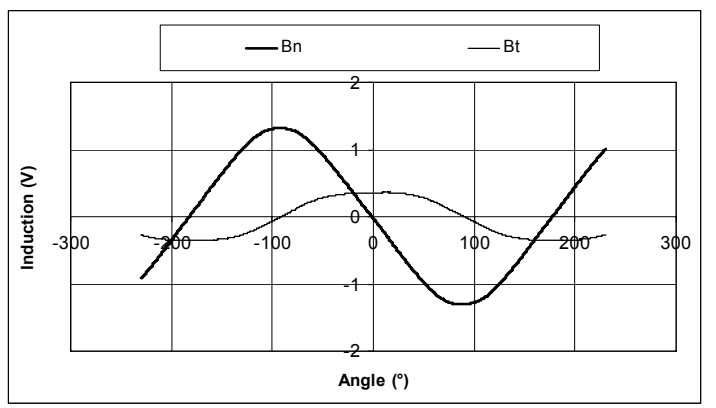

Figure 5 : Harmonic distortion of normal and tangential magnetic field components in a $360^{\circ}$ through shaft sensor

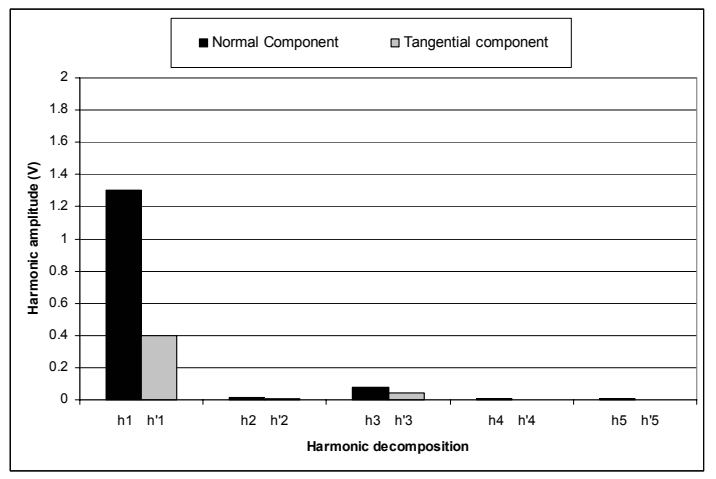

Figure 6 : Harmonic content on normal and tangential components

Considering that, MMT has built a solution [8] which is described hereafter (Figure 7). In that case, we use two ICs that are $90^{\circ}$ mechanically shifted. Each of them measures two components of the magnetic field, as for example the tangential and radial one in the figure.

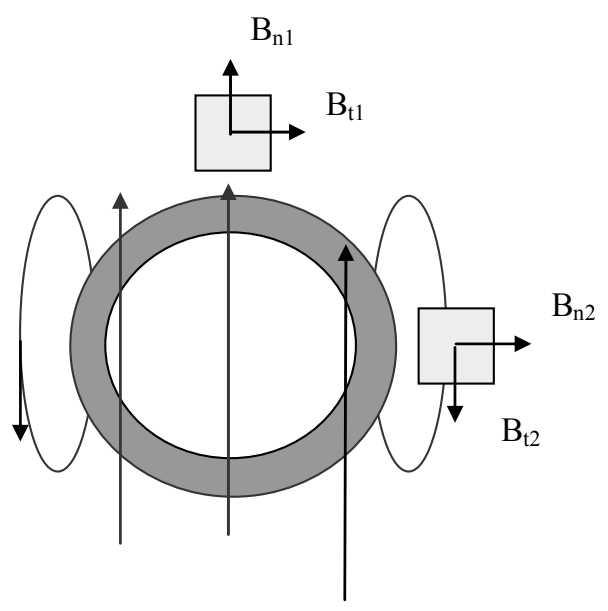

Let $B_{n 1}$ and $B_{t 1}$ (respectively $B_{n 2}$ and $B_{t} 2$ ) be the field components measured by the first (respectively the second) IC. If we suppose that the diametrical magnetization is not perfect and that the third harmonics is the only contributing perturbation, the magnetic field components $B_{n 1}$ and $B_{t 1}$ along the angular position $\theta$ can be written as follows :

$$
\begin{aligned}
& B_{n 1}=h_{1} \cdot \cos (\theta)+h_{3} \cdot \cos (3 \theta) \\
& B_{t 1}=h_{1}^{\prime} \cdot \sin (\theta)+h^{\prime} \cdot \sin (3 \theta)
\end{aligned}
$$

Where $h_{1}$ and $h_{3}$ are the amplitudes related to the fundamental and third harmonics.

Considering that the ICs are $90^{\circ}$ mechanically shifted, one can also write :

Figure 7 : Setup for improved accuracy sensor

$$
\begin{aligned}
& B_{n 2}=h_{1} \cdot \cos \left(\theta+\frac{\pi}{2}\right)+h_{3} \cdot \cos \left(3\left(\theta+\frac{\pi}{2}\right)\right) \\
& B_{t 2}=h_{1}^{\prime} \cdot \sin \left(\theta+\frac{\pi}{2}\right)+h_{3}^{\prime} \cdot \sin \left(3\left(\theta+\frac{\pi}{2}\right)\right)
\end{aligned}
$$

or

$$
\begin{aligned}
& B_{n 2}=-h_{1} \cdot \sin (\theta)+h_{3} \cdot \sin (3 \theta) \\
& B_{t 2}=h_{1}^{\prime} \cdot \cos (\theta)-h^{\prime} \cdot \cos (3 \theta)
\end{aligned}
$$

Let us now apply the following combinations:

$$
\begin{aligned}
& B_{n}=B_{n 1}+B_{t 2} \\
& B_{t}=B_{t 1}-B_{n 2}
\end{aligned}
$$

(4) which yield

$$
\begin{aligned}
& B_{n}=\left(h_{1}+h_{1}^{\prime}\right) \cdot \cos (\theta)+\left(h_{3}-h_{3}^{\prime}\right) \cdot \cos (3 \theta) \\
& B_{t}=\left(h_{1}+h_{1}^{\prime}\right) \cdot \sin (\theta)+\left(h_{3}^{\prime}-h_{3}\right) \cdot \sin (3 \theta)
\end{aligned}
$$

As $h_{3}$ and $h_{3}$ are small but also generally in the same order of magnitude (Figure 6), their difference can be neglected. Therefore, we have:

$$
\begin{aligned}
& B_{n} \approx\left(h_{1}+h_{1}^{\prime}\right) \cdot \cos (\theta) \\
& B_{t} \approx\left(h_{1}+h_{1}^{\prime}\right) \cdot \sin (\theta)
\end{aligned}
$$


Which means that the position can be directly obtained by calculating Arctan $(\mathrm{Bt} / \mathrm{Bn})$ with a good approximation. One can notice that this also means that no gain adjustment factor is necessary here.

The following figures show typical measurement of $B_{n 1}$ and $B_{t 1}$ (Figure 8 ) and then computed $B_{n}$ and $B_{t}$ signals (Figure 9), highlighting their equal amplitudes and sinusoisal shape as compared with $B_{n 1}$ and $B_{t 1}$, and then the non-linearity obtained with a sample (Figure 10). One can notice that a non-linearity of $+/-0.2 \%$ of the full stroke is now reached.

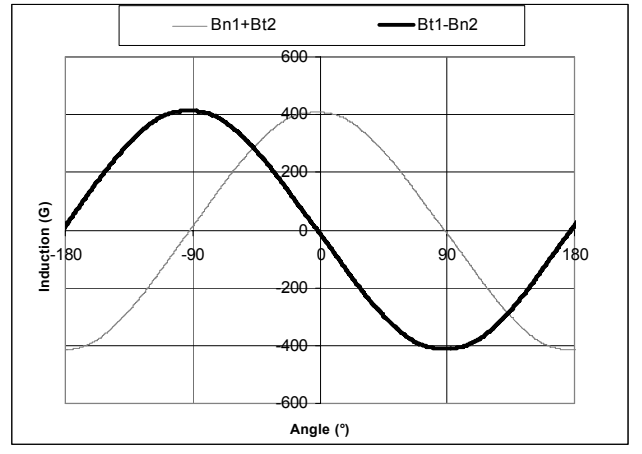

Figure 9 : Measured signals combination

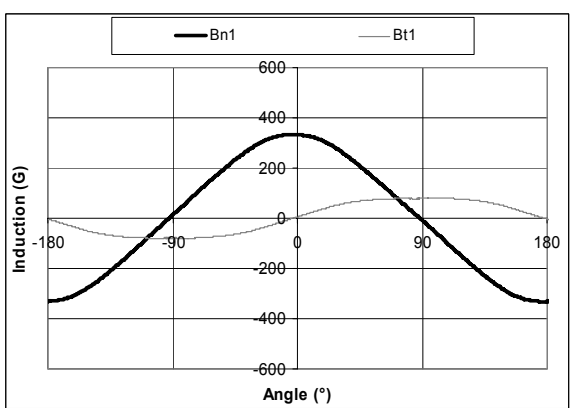

Figure 8 : measured $B_{n 1}$ and $B_{t 1}$ components

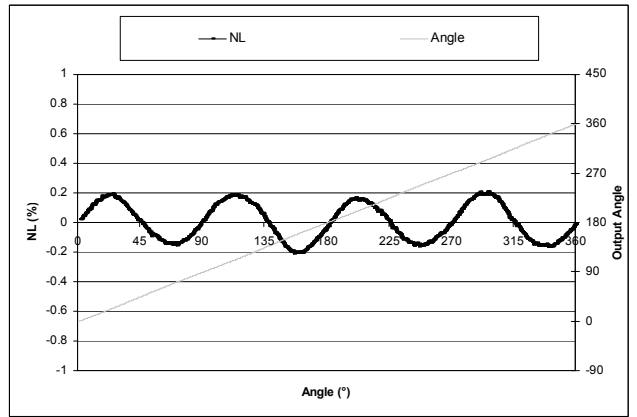

Figures 10 : Non-linearity calculated after signal combination

\section{External magnetic field cancellation}

Magnetic field lines generated by the presence of an external permanent magnet or a current source (wire with high current as for example a starter cable in a car) are a common source of error in such sensors where there is no magnetic circuit providing a natural shielding.

In that scope, MMT developped a specific know-how in the design of appropriate "local shielding" circuits to reduce external magnetic field effects. However, these solutions are built on a case-by-case basis and focus more on reducing than on canceling the perturbating field. Therefore, it is also interesting to look for a method that would be generic but also proposing a full theoretical cancellation of external fields.

The use of signal combination is a known way to achieve this in some sensors [9]. Considering that our higher accuracy sensor principle relies on signal combination, it is interesting to check how external fields can be handled. Let us consider again the sensor of Figure 7 submitted to an external and homogeneous magnetic field in its plane (Figure 11), a component in the third direction being not influent on the sensor. Let this field be defined by two components Bx_ext and By_ext.
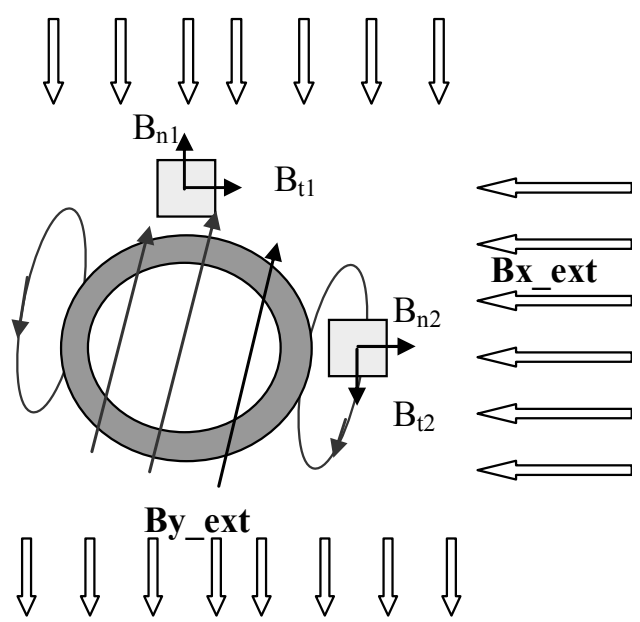

Figure 11 : Decomposition of planar homogeneous external magnetic field

Let us write again field components measured by the ICs. For convenience, third harmonics of magnetization will not be considered. In a given position $\theta$ we have :

$$
\begin{aligned}
& B_{n 1}=h_{1} \cdot \cos (\theta)-B y_{-} \text {ext } \\
& B_{t 1}=h_{1} \cdot \sin (\theta)-B x_{-} \text {ext }
\end{aligned}
$$

or

$$
\begin{aligned}
& B_{n 2}=h_{1} \cdot \cos \left(\theta+\frac{\pi}{2}\right)-B x_{-} \text {ext } \\
& B_{t 2}=h_{1}^{\prime} \cdot \sin \left(\theta+\frac{\pi}{2}\right)+B y_{-} \text {ext }
\end{aligned}
$$

Applying again the signal combination of Equation 4, we get : 


$$
\begin{gathered}
B_{n}=\left(h_{1}+h_{1}^{\prime}\right) \cdot \cos (\theta) \\
B_{t}=\left(h_{1}+h_{1}^{\prime}\right) \cdot \sin (\theta)
\end{gathered}
$$

Therefore, we have shown that using this combination, we can at the same time compensate the third harmonics magnetization error and the effect of an homogeneous external magnetic field.

Let us now provide some practical results with a sensor prototyple placed in a field generating coil as per Figure 12. One can notice (Figure 13) that even under a very high magnetic field of $120 \mathrm{G}$ (which means for example $1500 \mathrm{Amps}$ at $2.5 \mathrm{~cm}$ from the sensitive elements), the accuracy stays in the range of $+/-1^{\circ}$ or $+/-0.3 \%$ of the full stroke.

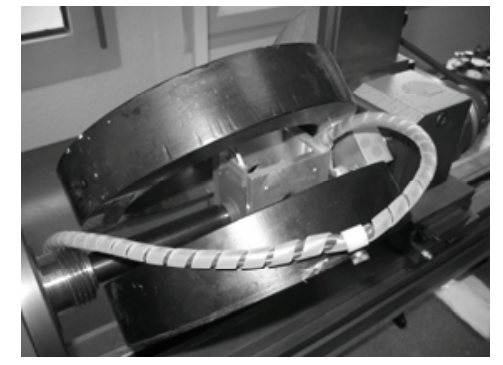

Figure 12 : measurement setup using generating coils

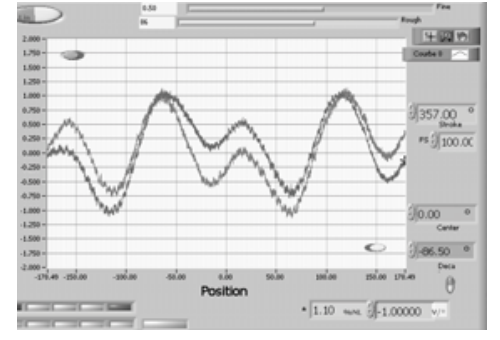

Figure 13 : Non Linearity (in degrees) with and without external magnetic field (120G)

Of course, this method depends on how homogeneous the field is. First of all, one has to notice that typical sensor sizes are small (diameter in the range 10 to $50 \mathrm{~mm}$ ), which induces small distances between the measuring points (typically a few millimeters). Then, sources are in general reasonably far from the sensor. Typical examples are a starter cable located a few tens of millimeters away from the sensor package or coils of a brushless DC motor that generate a field which is homogeneous at the scale of the sensor.

\section{Absolute multi-turn sensor concept}

Some specific sensor functions require an absolute output on multiple turns. Typically, automotive steering angle sensor may need this feature on the complete stroke of the steering wheel (for example +/- 2 turns). This specific application also requires the shaft (steering column) to pass through. So therefore it is a direct application case for our $360^{\circ}$ through shaft sensor solutions mentionned here above.

A first solution to provide a multi-turn output would be to associate our $360^{\circ}$ sensor with an epicyclic geartrain (Figure 14 ). However, the problem is that this sensor in its standard configuration with a typical non-linearity of $+/$ $0.5 \%$ of the full stroke would have a poor accuracy which is non compatible with the application requirements (typically $+/-7.2^{\circ}$ for $+/-2$ turns).

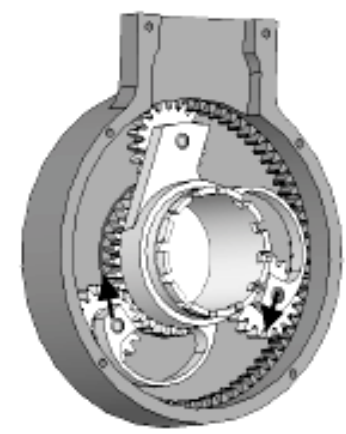

Figure 14 : Absolute multi-turn sensor using an epicyclic geartrain

An other solution would be to associate our improved sensor concept with the signal combination principle introduced at previous paragraph. Then, a typical non linearity of $+/-0.2 \%$ would yield an accuracy of $+/-2.9^{\circ}$ which is still unsufficient in some cases. Other solutions have been proposed by various companies (see for example 10). In such case, the rotary shaft is driving two pinions having a slightly different number of teeth (for example differing from 1 unit). Each pinion is coupled with a permanent magnet whose rotation is detected by a magnetosensitive IC. This yields two saw-tooth signal output whose phase shift is increasing over the number of turns. A specific algorithm is then used to deduce the absolute position from the difference between the two signals. The drawback of this solution is that due to the fact it uses a mathematical substraction is it very sensitive to any error or mechanical shift on each sensor. As a consequence, the algorithm has to be quite complex to reach a good accuracy.

In this paper, we propose an other solution providing a direct signal output without specific signal processing. The idea here is to provide a dual output by combining two $360^{\circ}$ sensor and a gear system [11]. One sensor (2) uses our standard $360^{\circ}$ through shaft solution and provides a fine output on a single turn while the other (1) is associated to an off-the-shaft gear train having a gear ratio in the range of the application's number of turns. The output of this sensor is absolute over the number of turns, but with a slightly worse accuracy. The role of this signal is to discriminate the actual turn while the fine output signal provides the accurate position inside the turn. 
This sensor may be of various type and for example use an end of shaft magnet with any IC using aforementioned field angle rotation detection. Figure 15 shows some embodiments with various gear concepts while Figure 16 provides prototype design and measurement result.
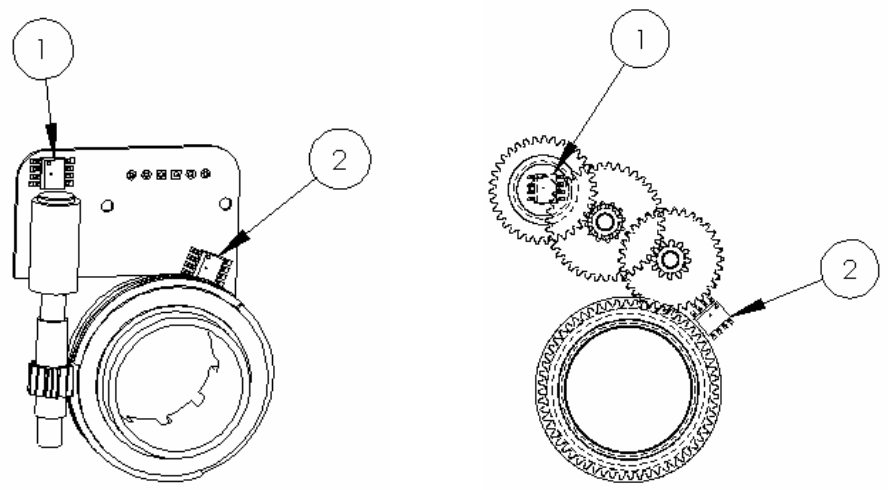

Figure 15: Two variants of absolute multi-turn sensor with dual output (sensor (1) and (2))
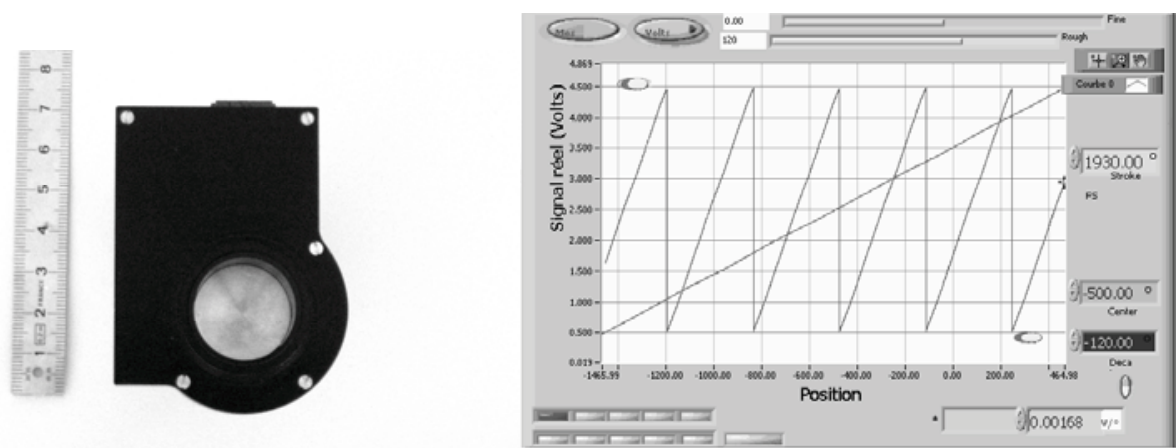

Figure 16 : Prototype example and measured dual output signal

\section{Conclusion}

In this paper, we have introduced a generic, efficient and simple solution to achieve high accuracies in a through shaft $360^{\circ}$ sensor. Further steps will be now to apply it to specific applications like steering wheel sensor for ESP or electric motor shaft absolute position sensor.

We also have introduced solutions for absolute multi-turn sensing. In that area, next steps will be to study integration with torque sensor for combined functions related to steering system and to pursue on the path toward a simple, reliable (and possibly contactless) multi-turn sensor.

\section{References}

[1] http://www.melexis.com/Assets/MLX90316 DataSheet 4834.aspx.

[2] http://www.austriamicrosystems.com/eng/Products/Magnetic-Encoders/Rotary-Encoders/AS5145.

[3] http://www.infineon.com - TLE5011 data sheet.

[4] 'Magnetic position and speed sensor having a Hall probe', MMT European patent EP0514530.

[5] 'Capteur rotatif $360^{\circ}$ notamment pour colonne de direction automobile', MMT French patent FR2893410.

[6] 'Capteur de position à direction d'aimantation variable et procédé de réalisation', MMT French patent FR2898189.

[7] N. Jerance et al. 'Through-shaft contactless magnetic sensor with a stroke up to 360', Sensor+Test Conference 2007.

[8] 'Capteur de position magnétique angulaire ou linéaire présentant une insensibilité aux champs extérieurs', MMT French patent application, not published yet.

[9] 'Capteur de position présentant une insensibilité aux champs extérieurs et aux excentrations', MMT French patent FR2809808.

[10] 'Method to determine absolute position with displacement and angle transducers; involves using two mechanically coupled sensors with output signals with numbers of periods different by one', US patent US6,466,889.

[11] 'Capteur magnétique sans contact de position absolue multitour à arbre traversant', MMT French patent application, not published yet. 